\title{
Investigation of Thermal Performance of Mold Copper Plate in Slab Continuous Casting
}

\author{
Xingzhong Zhang ${ }^{*}, 1$, Zhengyi Jiang ${ }^{2}$, Qiang Zhang ${ }^{3}$ and Chunxia Dou ${ }^{4}$ \\ ${ }^{I}$ College of Mechanical Engineering, Yanshan University, Qinhuangdao, 066004 P.R. China \\ ${ }^{2}$ School of Mechanical, Materials and Mechatronic Engineering, University of Wollongong, Wollongong NSW 2522, \\ Australia \\ ${ }^{3}$ Shanxi Rolling Equipment Company, Xi'an, 711711 P.R. China \\ ${ }^{4}$ College of Electrical Engineering, Yanshan University, Qinhuangdao, 066004 P.R. China
}

\begin{abstract}
Based on the cooling parameters of the mold copper plate for slab continuous casting in a steelmaking plant, the three-dimensional calculating model of the copper plate was established, and three-dimensional distributions of temperature, thermal stress and strain were simulated numerically by using finite element method (FEM). The maximum deformation of the mold copper plate, the highest temperature and thermal stress were obtained. This research is useful for the structure design of the mold.
\end{abstract}

Keywords: Slab continuous casting, mold copper plate, temperature, thermal stress, thermal deformation.

\section{INTRODUCTION}

Mold is the core part of continuous caster. Heat transfer effect of mold has a direct impact on continuous casting production process and internal quality of slab. In the cooling process, the mold not only carried out the heat transferred from liquid steel, but also supports static pressure from molten steel, and friction, etc. The mold copper plate (or called mold copper) works under an extremely poor condition which includes high temperature, friction between the slab and the mold, the mechanical and thermal stresses and the pressure of the cooling water. In order to make the mold work properly, the mold structure and the materials of the mold copper must be suitable. For example, to make the molten steel cooled efficiently, the mold copper should contact perfectly with the slab surface, and there should be no gap between them. This demands the mold should be tapered, and the taper design should take the deformation of the mold copper into account. The cooling water pressure is very high, so the thermal deformation of the mold copper should not be big enough to make the cooling water leak. The mold copper should have a better resistance to abrasion. The inner surface of the mold copper is heated by the slab, and the outside surface is cooled by the cooling water, so the temperature difference would cause the thermal stress and deformation. The deformation and thermal stress should be taken into account when the mold structure, such as the taper, seam size and the strength are designed.

Yang et al. had done some research works about threedimensional heat transfer analysis on thin slab continuous

*Address correspondence to this author at the College of Mechanical Engineering, Yanshan University, Qinhuangdao 066004, P. R. China; Tel: +86-335-8057031 (Office), +86-13933580278 (Mobile); Fax: +86-3358074783; E-mails: zhangxzh@ysu.edu.cn, zhangxingzh@sina.com casting mold copper [1]. Feng et al. had simulated the temperature field on the slab continuous casting mold copper with finite element method (FEM) [2]. In accordance with the result, they re-allocated the water seam. Liu and Zhu conducted the research on analysis of thermal elastoplastic behavior of continuous casting slab mold [3]. Wang et al. optimized the mold taper in high speed casting of steel billets [4]. Long and Peng analyzed the thermal deformation of mold copper by using FEM [5].

In this paper, based on the method of coupling the mold copper heat transfer with the stress/strain and deformation, the temperature field, stress/strain and thermal deformation of the mold copper during slab continuous casting process were successfully analyzed by using FEM.

\section{CALCULATING MODEL AND PARAMETERS}

\subsection{Finite Element Model}

Before modeling, some assumptions are made as following without causing large calculation error.

1. Ignore the impact of molten steel fluctuation on the mold copper near the meniscus.

2. Ignore the impact of mold oscillation on the mold copper.

3. Consider that the contact between the mold copper and the backplane is closely. Ignore the impact of bolts on the mold copper.

4. Consider that the mold copper is isotropic.

Because the distribution of water seam is symmetric, half of the cross-sectional area between two bolts is taken as the computational domain. The mesh used in FE simulation is shown in Fig. (1). 


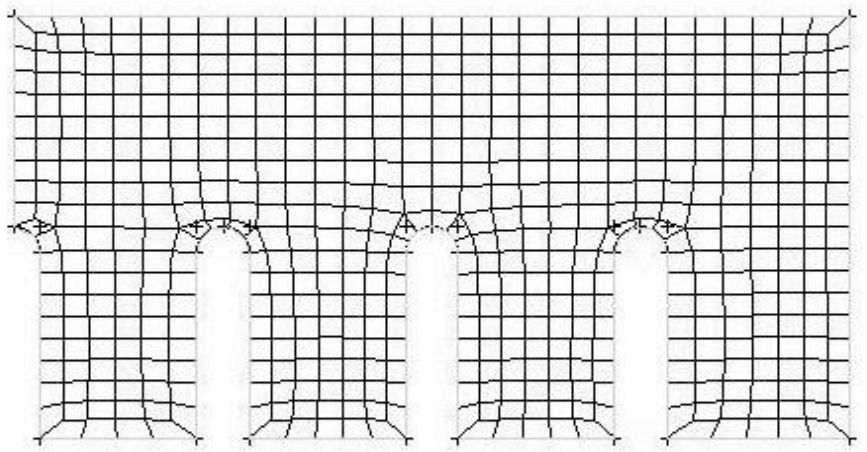

Fig. (1). Mesh of mold copper.

\subsection{Parameters of the Mold Copper}

The structural parameters of the mold copper are shown in Table 1. The material of the mold copper is chromium zirconium - copper alloy, which the mass fraction of three elements is:

$$
\text { Cr: } 0.5-1.5 \% ; \mathrm{Zr}: 0.05-0.3 \% ; \mathrm{Cu}: 98 \%
$$

Table 1. Structural Parameters of the Mold Copper

\begin{tabular}{|c|c|c|}
\hline Items & Units & Values \\
\hline \hline Mold copper thickness & $\mathrm{mm}$ & 40 \\
\hline Bolts spacing & $\mathrm{mm}$ & 160 \\
\hline Bolt type & & M30 \\
\hline Water seam spacing & $\mathrm{mm}$ & 20 \\
\hline Width of water seam & $\mathrm{mm}$ & 5 \\
\hline Depth of water seam & $\mathrm{mm}$ & 20 \\
\hline
\end{tabular}

The alloy not only has high hardness and strength, but also good thermal conductivity, wear and corrosion resistance. Its softening temperature is 500 degree, and the linear expansion coefficient is $1.7 \times 10^{-5} /$ degree. The thermal conductivity coefficient is a function of temperature which can be described as (1).

$k=317.75+0.1224 \times T-0.0001 \times T^{2}$

where the unit of temperature $T$ is degree. $k$ is the thermal conductivity coefficient, the unit is $\mathrm{W} /(\mathrm{m} \cdot$ degree $)$. The specific heat is $384 \mathrm{~J} /(\mathrm{kg} \cdot$ degree $)$. Young's modulus $E$ is 115 $\mathrm{GPa}$. Poisson ratio is 0.34 .

\section{HEAT TRANSFER AND MECHANICAL BOUND- ARY CONDITIONS}

To analyze the temperature, stress and deformation of the mold copper, the corresponding boundary conditions must be determined firstly.

\subsection{Inner Surface of the Mold Copper}

On the inner surface of the mold copper which contacts with the slab, the heat flux can be calculated using Eq. (2) [6], $q=2680-335 \sqrt{t}$

where $q$ is the heat flux, $\mathrm{KW} / \mathrm{m}^{2} . t$ is the time that the slab stays in the mold, and its unit is second, and $t=L / v$ (where $L$ is the distance from the calculating position to the meniscus, the unit is m. $v$ is the casting speed, in this study, $v$ is 0.9 $\mathrm{m} /$ minute).

\subsection{Surface of Water Seam}

On the surfaces of water seams which contact with the cooling water, the heat flux can be calculated using Eq. (3),

$q=h\left(T_{\text {copper }}-T_{\text {water }}\right)$

where $q$ is the heat flux, $\mathrm{W} / \mathrm{m}^{2} . T_{\text {copper }}$ is the temperature of the water seam surface which contacts with the cooling water, degree. $T_{\text {water }}$ is the temperature of the cooling water, degree. $h$ is the heat transfer coefficient between the mold copper and cooling water, $\mathrm{W} /\left(\mathrm{m}^{2} \cdot\right.$ degree $)$, and it can be calculated using Eq. (4) [7].

$$
h=0.023 \cdot\left(\frac{D \cdot v \cdot \rho}{\mu}\right)^{0.8} \cdot\left(\frac{C \cdot \mu}{\lambda}\right)^{0.4} \cdot \frac{\lambda}{D}
$$

where $D$ is the equivalent diameter of water seam, its unit is m. $v$ is the flow speed of cooling water, which equals 10.5 $\mathrm{m} / \mathrm{s} . \rho$ is the density of cooling water, $\mathrm{kg} / \mathrm{m}^{3} . \mu$ is the viscosity of cooling water, $\mathrm{kg} /(\mathrm{m} \cdot \mathrm{s}) . \lambda$ is the thermal conductivity coefficient of cooling water, $\mathrm{W} /(\mathrm{m} \cdot$ degree $) . C$ is the specific heat of cooling water, $\mathrm{J} /(\mathrm{kg} \cdot$ degree $)$.

\subsection{Surface of the Mold Copper Contacting with the Backplane}

On the surface of the mold copper contacting with the backplane, the heat flux can be calculated using Eq. (5).

$q=h_{a}\left(T_{b a c k}-T_{0}\right)$

where $T_{\text {back }}$ is the temperature of the mold copper surface which contacts with the backplane, degree. $T_{0}$ is the temperature of the backplane, degree. $h_{a}$ is the heat transfer coefficient between the mold copper and the backplane, which equals $10000 \mathrm{~W} /\left(\mathrm{m}^{2} \cdot\right.$ degree $)$, and the initial temperature of backplane is $T_{0}=20$ degree.

\subsection{Mechanical Boundary Conditions}

The backplane of the mold is considered as a rigid body, and its displacements at any direction equal zero. The mold copper is considered as elastomer, and at any bolt position the displacement of the mold copper is zero.

\section{ANALYSIS OF SIMULATION RESULTS}

\subsection{Temperature, Stress and Deformation Analysis of the Mold Copper at Meniscus}

The distributions of the temperature, thermal stress and deformation of the mold copper at meniscus are shown in Figs. (2-4). And at the middle of mold copper, along the thickness direction the distributions of the temperature, thermal stress and deformation are shown in Fig. (5-7). From these figures it can be seen that: 
1. The highest temperature at the inner surface of the mold copper is 218.8 degree. It is far away from the molten temperature. However, the high temperature will lower the hardness of the mold copper. So the mold copper here is easy worn down.

2. The temperature gradient near the inner surface is relatively large. Therefore, the thermal stress near the inner surface is greater than that of other parts.

3. Due to the strong cooling effect of cooling water, the rapid temperature change results in the stress concentration near the bottom of the water seam.

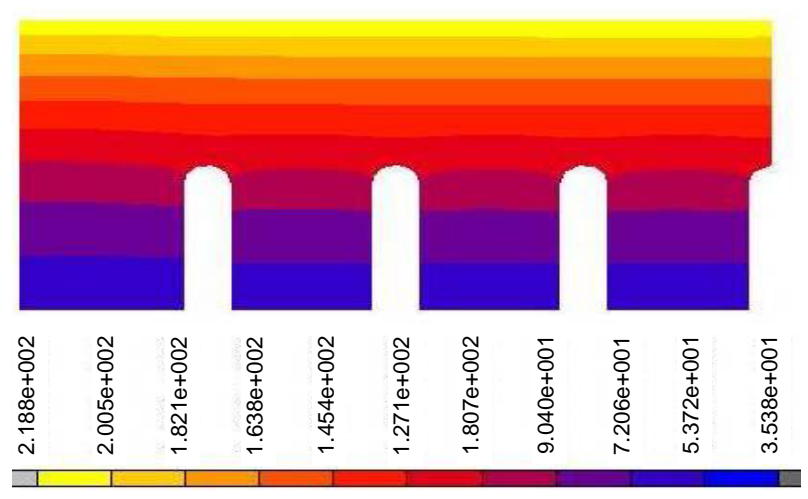

Fig. (2). Temperature distribution of the mold copper.

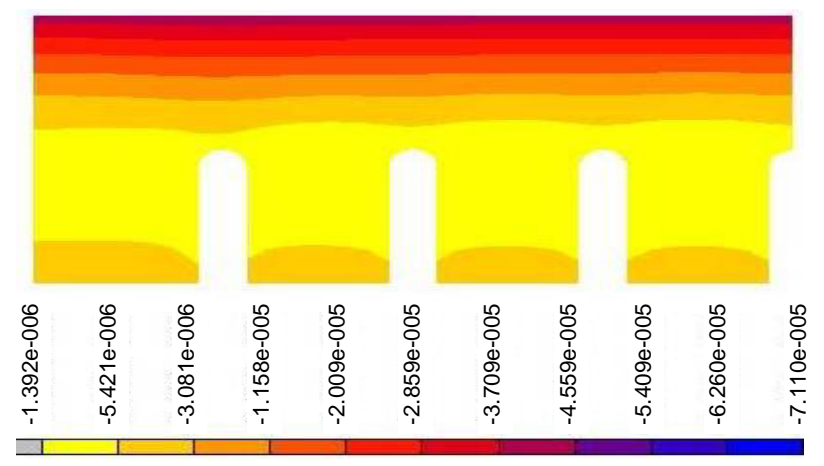

Fig. (3). Deformation distribution of the mold copper.

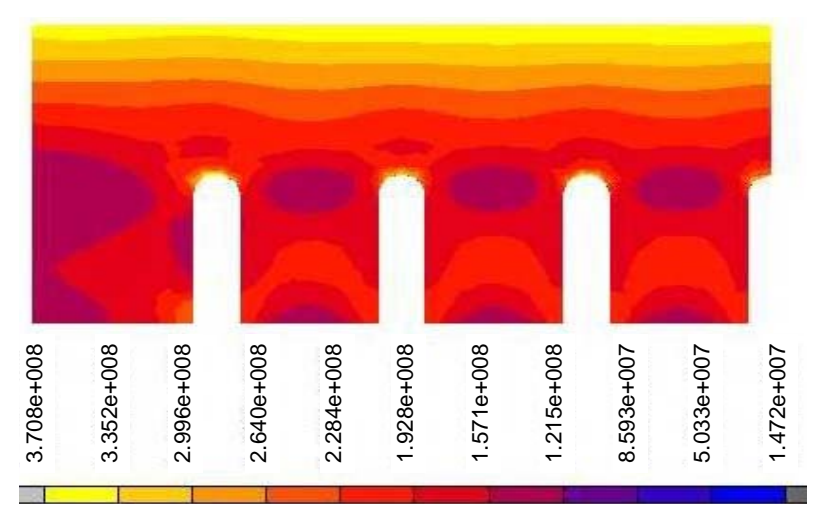

Fig. (4). Thermal stress distribution of the mold copper.

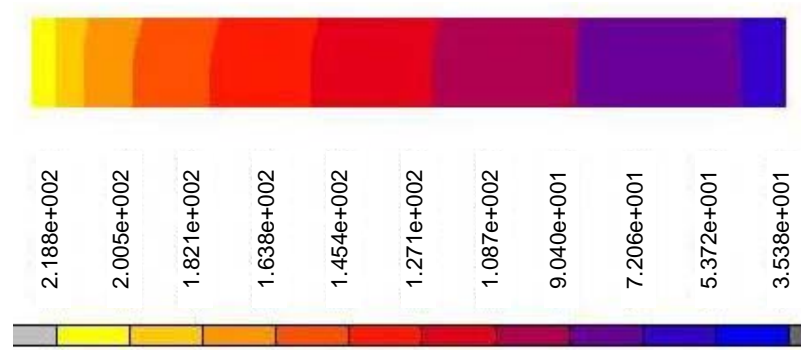

Fig. (5). Temperature distribution at the middle of mold copper.
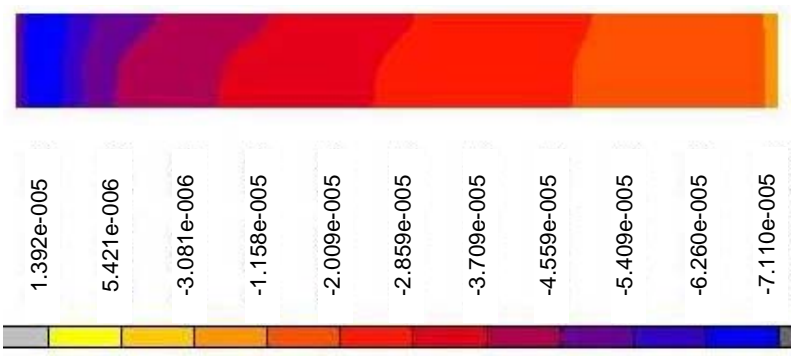

Fig. (6). Deformation distribution at the middle of mold copper.
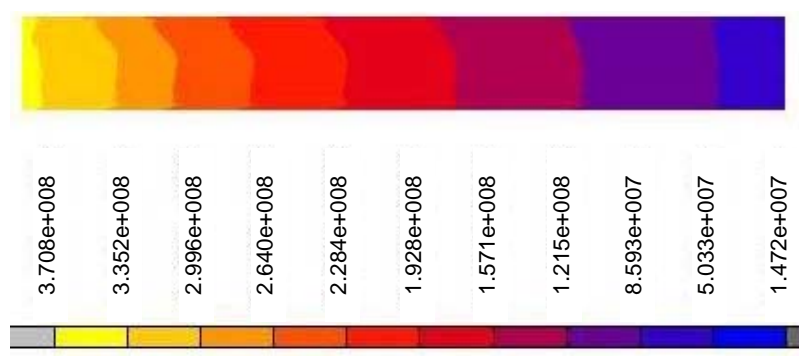

Fig. (7). Thermal stress distribution at the middle of mold copper.

\subsection{Curves of Temperature, Stress and Deformation}

Along the casting direction, the temperature, the thermal deformation and the thermal stress distributions at the middle position of the inner surface of the mold are shown in Fig. (8) to Fig. (10).

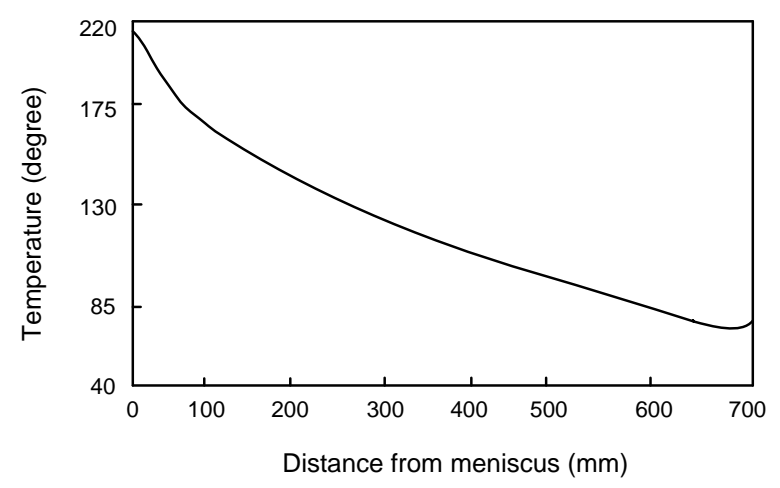

Fig. (8). The inner surface temperature distribution.

It can be seen form Fig. (8) that the temperature of the mold copper changes in accordance with the heat flux, which was expressed in Eq. (2). The highest temperature is at the meniscus, and along the casting direction, the inner surface 
temperature falls sharply. But at the position of $40 \mathrm{~mm}$ from mould outlet, it begins to return a little. This is due to there are no water seams near the bottom of the mold copper. The poor heat transfer results in the temperature increase.

It can be seen from Fig. (9) that the largest deformation is about $0.07 \mathrm{~mm}$, which appears in the vicinity of meniscus. The deformation decreases rapidly with the decrease of the inner surface temperature of the mold copper.

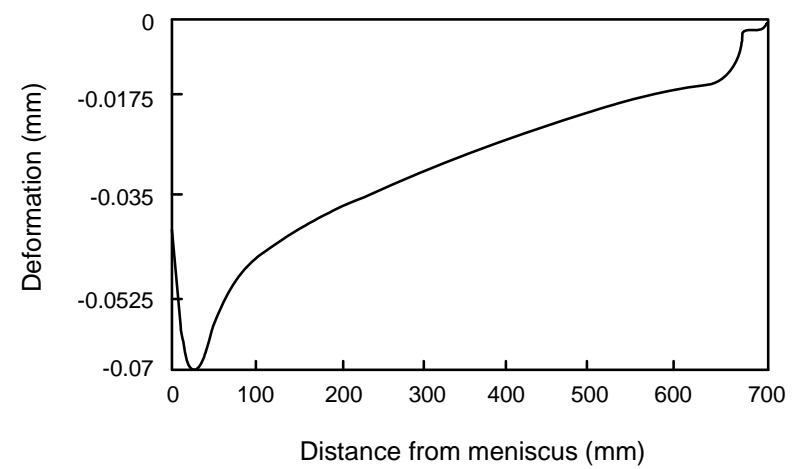

Fig. (9). The deformation distribution.

It can be seen from Fig. (10) that the thermal stress decreases rapidly along the casting direction. The biggest thermal stress appears at the meniscus, and its value is about $360 \mathrm{MPa}$, and at the outlet of the mold, the thermal stress is lower. Because the high temperature of the mold copper lowers its hardness near the meniscus, the bigger thermal stress, the oscillation of mold, the fluctuation of the molten steel surface together with the erosion effect of the powder would make the mold copper surface near the meniscus more erosive and abrasive.

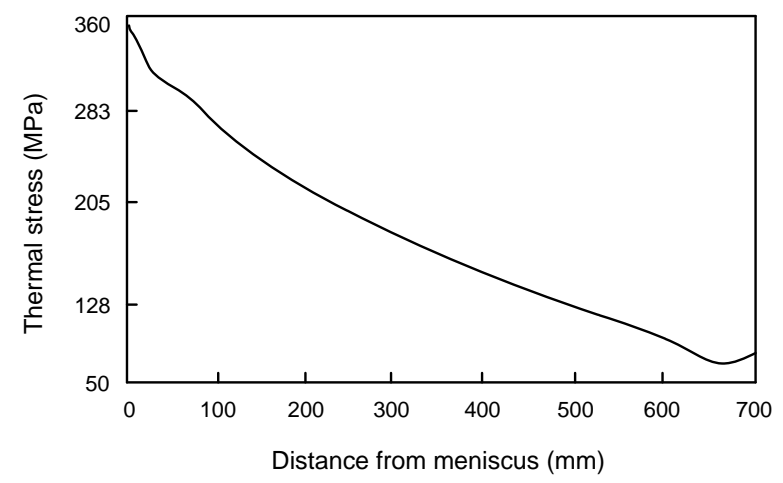

\section{CONCLUSION}

The highest temperature of the mold copper appears on the inner surface at the meniscus position. The inner surface temperature of the mold copper declines rapidly along the casting direction, and it increases a little near the outlet of the mold. The surface temperature of water seam changes little along the casting direction, and the biggest temperature difference from the top to the bottom is not bigger than 10 degree.

The maximum thermal deformation of the mold copper appears at position $30 \mathrm{~mm}$ downwards from the meniscus. The deformation is not big enough to cause the leaking problem of the cooling water.

The thermal stress on the inner surface of the mold copper is bigger than that of the water seam surface. There is a little stress concentration at the bottom of the water seam, but it is not severe enough to result in cracks.

\section{ACKNOWLEDGEMENT}

The authors thank the National Science Foundation of China for supporting the current research under grant 60874023 .

\section{REFERENCES}

[1] G. Yang, B. K. Li, and Y. Yu, "There dimensional heat transfer of cooling copper plate of thin slab continuous casting mold", Acta Metal. Sin., vol. 43, pp. 332-336, March 2007.

[2] K. Feng, Z. W. Han, and J. H. Mao, "Finite element simulation of temperature field of mold copper plate for continuous casting", Foundry Technol., vol. 28, pp. 135-138, January 2007.

[3] X. D. Liu, and M. Y. Zhu, "An analysis of thermal elastoplastio behavior of continuous casting slab mold", Acta Metal. Sin., vol. 42, pp. 1137-1142, November 2006.

[4] J. G. Wang, B. F. Wang, and F. Dong, "Optimization of the mold taper in high speed casting of steel billets", Continuous Casting, pp. 10-14, April 2009.

[5] X. S. Long, and X. H. Peng, "FEM analysis of thermal and deformation of mold copper", Iron Steel, vol. 41, pp. 35-38, October 2006.

[6] M. Y. Liu, P. Li, and X. J. Luan, Slab Continuous Caster, Beijing: Mechanical Industry Press, 1990.

[7] J. Q. Sun, and Q. G. Liu, Heat Transfer, Harbin: Northeat Forestry Univeristy Press, 1997.

Fig. (10). The thermal stress distribution. 